IMA Journal of Applied Mathematics (2018) Page 1 of 15 doi:10.1093/imamat/xxx000

\title{
On the formation of small-time curvature singularities in vortex sheets
}

\author{
Michael A. Page* \\ School of Mathematical Sciences, Monash University, Victoria 3800, Australia \\ *Michael.Page@monash.edu \\ AND \\ STEPHEN J. COWLEY \\ DAMTP, University of Cambridge, Wilberforce Road, Cambridge CB3 OWA, UK
}

[Received on 25 April 2018]

\begin{abstract}
The Kelvin-Helmholtz model for the evolution of an infinitesimally thin vortex sheet in an inviscid fluid is mathematically ill-posed for general classes of initial conditions. However, if the initial data, say imposed at $t=0$, is in a certain class of analytic functions then the problem is well-posed for a finite time until a singularity forms, say at $t=t_{s}$, on the vortex-sheet interface, e.g. as illustrated by Moore (1979). However, if the problem is analytically continued into the complex plane, then the singularity, or singularities, exist for $t<t_{s}$ away from the physical real axis. More specifically, Cowley et al. (1999) found that for a class of analytic initial conditions, singularities can form in the complex plane at $t=0+$. They posed asymptotic expansions in the neighbourhood of these singularities for $0<t \ll 1$, and found numerical solutions to the governing similarity differential equations. In this paper we obtain new exact solutions to these equations, show that the singularities always correspond to local $\frac{3}{2}$-power singularities, and determine both the number and precise locations of all branch points. Further, our analytical approach can be extended to a more general class of initial conditions. These new exact solutions can assist in resolving the small-time behaviour for the numerical solution of the Birkhoff-Rott equations.
\end{abstract}

Keywords: Kelvin-Helmholtz instability, vortex-sheet singularities.

2010 Math Subject Classification: 76E17, 34M35

\section{Introduction}

A fundamental feature of shear flows at high Reynolds numbers is the Kelvin-Helmholtz instability of a vortex sheet. A key aspect of this instability on an infinitesimally thin vortex sheet is that the problem is ill-posed, since linear sinusoidal disturbances with a wavenumber $k$ have a growth rate proportional to $k$, e.g. see Saffman \& Baker (1979). However, the vortex-sheet problem is well-posed for a finite time if the initial data lies in a certain class of analytic functions, as noted by Sulem et al. (1981), Caflisch \& Orellana (1986) and Duchon \& Robert (1988). Indeed, in a seminal paper, Moore (1979) showed that the cumulative influence of nonlinear effects on the evolution of a small disturbance that is initially sinusoidal, is such that a local $\frac{3}{2}$-power curvature singularity can develop after a finite time.

A characteristic of numerical solutions for a range of initial conditions, say imposed at $t=0$, was the formation of $\frac{3}{2}$-power curvature singularities, rather than other powers, e.g. Meiron et al. (1982), Krasny (1986) and Pugh (1989). In an attempt to explain this, Cowley et al. (1999), hereinafter CBT, used analytic continuation in the complex circulation plane based on the formulation of Moore (1985) and Caflisch \& Orellana (1986). In particular, CBT examined the small-time asymptotic solution at key points in the complex plane away from the real physical axis and showed that, even for analytic 
initial conditions, singularities could form at time $t=0+$ at isolated points. Once these singularities had formed they could then move with time around the complex plane without changing their strength, until at some finite time, say $t=t_{s}$, one or more hit the real axis; at that time the physical vortex-sheet problem would have a finite-time $\frac{3}{2}$-power curvature singularity. The character of the physical $\frac{3}{2}$-power curvature singularity at $t=t_{s}$ is therefore apparently fixed by the form of the one or more singularities that develop spontaneously at $t=0+$ away from the real physical axis.

CBT determined the nature of the spontaneous singularities by deriving asymptotic series solutions for $0<t \ll 1$ for one specific case, and two generic cases. For the generic cases, the solutions have similarity form and are governed by ordinary differential equations in the complex plane. The form of the singularities of these differential equations is what determines the form of the singularities of the vortex-sheet problem. CBT showed that a $\frac{3}{2}$-power singularity was consistent with the differential equations, and confirmed the existence of $\frac{3}{2}$-power singularities by solving the equations numerically. However, tracking the branch cuts numerically was difficult, and an analytical solution of the equations would be far more satisfactory. We present such an analytical solution here.

In $\S 2$ below the context of this new analytical solution is outlined, following the approach of CBT and earlier work. This is illustrated in $\S 3$ on the first singular case considered by CBT, where the governing equations of the small-time similarity solution are restated and solved analytically. To obtain this exact solution it is necessary to change independent variable but a prudent choice enables the solution to be expressed in a readily-interpretable form. The properties of this solution are then examined, in particular in comparison to the findings of CBT.

In $\S 4$ this exact analytical solution is extended to a broader class of initial conditions for which the similarity solution matches at higher than second order. Broadly the same features remain present but with a different number of singular points and branch cuts.

The key implications of these results are discussed in $\S 5$, in particular confirmation by analytical means of the locations of all of the branch-point singularities in the small-time similarity solution, and that the solution has a local $\frac{3}{2}$-power behaviour near those points. The existence of an analytical initial condition should also assist in the accurate numerical solution of the problem in the full complex plane.

\section{Formulation}

In describing the evolution of an interface on the approach to a singularity at a finite time, a similar formulation to that used by Caflisch \& Orellana (1986) and CBT is used here. In particular, the position $(x, y)$ of a vortex sheet is represented parametrically in terms of a corresponding complex-valued function $z(\xi, t)=\xi+s(\xi, t)$, where $s$ is periodic in terms of the real variable $\xi$, for example over $[0,2 \pi]$. The evolution of the position of the sheet, and hence $s(\xi, t)$, can be determined from the Birkhoff-Rott integro-differential equation (see for example Birkhoff (1962)). To help determine $s(\xi, t)$ the parameter $\xi$ is extended into the complex plane. CBT outline in more detail a suitable process for this, introducing a second complex-valued function $s^{*}$ with

$$
s^{*}(\xi, t)=\overline{s(\bar{\xi}, t)}
$$

where the unknowns $s$ and $s^{*}$ are determined by evaluating the coupled partial differential equations

$$
\frac{\partial s^{*}}{\partial t}=\frac{\sigma_{\xi}-s_{\xi}}{2\left(1+s_{\xi}\right)}+J(\xi, t) \quad \text { and } \quad \frac{\partial s}{\partial t}=-\frac{\sigma_{\xi}-s_{\xi}^{*}}{2\left(1+s_{\xi}^{*}\right)}+K(\xi, t) .
$$

Here $\sigma(\xi)$ is a time-independent real-valued function that represents the periodic part of the normalised circulation $\Gamma(\xi)=\xi+\sigma(\xi)$ across the sheet, and $J(\xi, t)$ and $K(\xi, t)$ are integrals in the complex plane 
which here are assumed to be analytic for all $(\xi, t)$ in the region of interest (and in particular close to $\left(\xi_{0}, 0\right)$ below).

The integration of (2.1) is commenced from an initial time $t=0$, say, at which $s_{0}(\xi)=s(\xi, 0)$ is given. For simplicity it will be assumed here that both $\sigma(\xi)$ and $s_{0}(\xi)$ are analytic functions at and near $\xi_{0}$ - even though that constraint may not be strictly necessary. Details of the other relevant assumptions and constraints are provided in CBT.

A special case of this problem was identified by Meiron et al. (1982), by taking $s_{0}(\xi)=0$ and $\sigma(\xi)=\varepsilon \sin (\xi)$ when $\varepsilon \ll 1$. Using a series solution, following Moore (1979), they found that for small times $0<t \ll 1$, singularities formed in $s$ and $s^{*}$ at large values of $|\operatorname{Im}(\xi)|$, i.e. $\xi_{0 \pm} \sim \pm i \ln (2 / \varepsilon t)$. CBT extended this result to $\varepsilon=O(1)$, using asymptotic analysis and demonstrated that $\frac{3}{2}$-power singularities in $s$ and $s^{*}$ formed spontaneously at $t=0+$ at $\xi_{0 \pm}$, which then moved towards the real axis as $t$ increased until they collided on that axis and resulted in a physical curvature singularity.

CBT analyzed a broader set of initial conditions, in particular those with $s_{0}$ non-zero, and proposed that, more generally, $s$ and $s^{*}$ could form $\frac{3}{2}$-power singularities 'spontaneously' at $t=0+$ in the complex plane (away from the real axis). These singularities could then move around the complex plane (without changing strength), until one or more intersected with the real $\xi$ axis generating a physical curvature singularity. CBT's analysis was based on a small-time asymptotic solution and covered two cases: specifically they argued that singularities can form when either one or both of the $\xi$-derivatives $s_{0 \xi}(\xi)$ and/or $s_{0 \xi}^{*}(\xi)$ were equal to -1 at some point $\xi_{0}$ in the complex $\xi$-plane. Similarity solutions were developed close to those points. However, CBT were unable to solve the coupled nonlinear ordinary differential equations (ODEs) that described the local form of the developing singularities, and hence resorted to numerical means to indicate the properties of the solutions. Although they were able to do that with some confidence, a better approach would have been to find exact solutions of the ODEs, which is what we do in this paper. These exact solutions clarify the developing solutions for small $t$.

\section{Exact solution for singularity formation: Case 1}

Following the approach of CBT, near a point $\xi=\xi_{0}$ such that $s_{0 \xi}\left(\xi_{0}\right)=-1$ but $s_{0 \xi}^{*}\left(\xi_{0}\right) \neq-1$, series solutions for $s(\xi, t)$ and $s^{*}(\xi, t)$ at small times $t$ are sought of the form

$$
\begin{aligned}
& s=s_{00}-\zeta+\frac{1}{2} s_{02} \zeta^{2}+\cdots+\left[\frac{1}{2}+K_{00}-\frac{\left(1+\sigma_{01}\right)}{2\left(1+s_{01}^{*}\right)}\right] t+\ldots, \\
& s^{*}=s_{00}^{*}+s_{01}^{*} \zeta+\frac{1}{2} s_{02}^{*} \zeta^{2}+\cdots+\left[\frac{\left(1+\sigma_{01}\right)}{2 s_{02} \zeta}+\ldots\right] t+\ldots,
\end{aligned}
$$

where $\zeta=\xi-\xi_{0}$ and the other constants are as defined by CBT (e.g. $K_{00}=K\left(\xi_{0}, 0\right)$ ). This expansion becomes 'disordered' once $\zeta \sim t / \zeta$, or for $\zeta=O\left(t^{1 / 2}\right)$, which suggests using a 'similarity' variable $\eta=\zeta / \omega t^{1 / 2}$ in terms of the constant

$$
\omega=\left(\frac{2\left(1+\sigma_{01}\right)}{s_{02}\left(1+s_{01}^{*}\right)}\right)^{1 / 2}
$$

CBT proposed a small-time expansion of $s$ and $s^{*}$ in terms of $\eta$ and $t$ with

$$
\begin{aligned}
& s=s_{00}-\eta \omega t^{1 / 2}+\left[\frac{1}{2}+K_{00}-\frac{\left(1+\sigma_{01}\right)}{2\left(1+s_{01}^{*}\right)} A(\eta)\right] t+\ldots, \\
& s^{*}=s_{00}^{*}+\left[-\eta+\left(1+s_{01}^{*}\right) B(\eta)\right] \omega t^{1 / 2}+\ldots,
\end{aligned}
$$


where $A$ and $B$ satisfy the coupled nonlinear first-order ordinary differential equations

$$
2 A-\eta A_{\eta}=-\frac{1}{B_{\eta}} \quad \text { and } \quad B-\eta B_{\eta}=\frac{1}{A_{\eta}}
$$

in terms any complex-valued variable $\eta$. As $\omega$ may be complex-valued in general, note that the axes of $\eta$ are not necessarily oriented in the same direction as those for $\xi$. Note also that the real $\xi$ axis, where the solution has a physical meaning, is effectively well outside the $\eta=O(1)$ domain.

To match with the initial conditions for $s$ and $s^{*}$, for large values of $\eta$ the third and second terms in (3.1a) and (3.1b), respectively, must match with the bracketed terms in (3.2a) and (3.2b). Consequently $A \sim \eta^{2}$ and $B \sim \eta$ for large $\eta$.

CBT were unable to find exact expressions for $A(\eta)$ and $B(\eta)$ so instead they sought to deduce some of their properties using numerical integration. In particular, assuming a four-fold symmetry of the solutions they demonstrated that both $A$ and $B$ appear to have (four) branch points, located at $\pm \eta_{0}$ and $\pm \bar{\eta}_{0}$, and that properties of these solutions are consistent with each having a $\frac{3}{2}$-power singularity at those branch points. By integrating from $\eta=L+i 0$ and $\eta=0+i L$, for large $L>0$, CBT identified no other singular points in the first quadrant, although they noted the presence of other singular points on "different Riemann sheets". For example, when instead integrating into the first quadrant from $\eta=0-i L$ they identified a singular point at $\eta_{1}=0.0829+i 0.1240$.

The analysis in this paper confirms the presence of up to eight branch points related to $\eta_{0}$ and $\eta_{1}$, and identifies their exact positions. In $\S 3.6$ it is shown that the number of branch points that are present will depend on how the branch cuts are configured, and in particular the assumed symmetry of the solutions. The analysis also confirms that there are no other singular points in $A$ or $B$ at a finite value of $\eta$.

\subsection{Integration of the coupled ODEs}

The key to determining an exact solution of the coupled nonlinear ODEs (3.4) is that they can be written in the equivalent form

$$
\left(2-\eta \frac{A_{\eta}}{A}\right)\left(\eta \frac{B_{\eta}}{B}\right)=-\frac{\eta}{A B} \quad \text { and } \quad \eta \frac{A_{\eta}}{A}\left(1-\eta \frac{B_{\eta}}{B}\right)=\frac{\eta}{A B}
$$

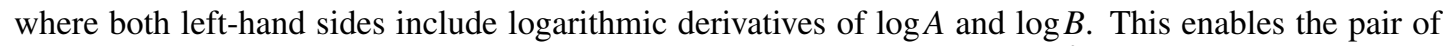
equations to be rewritten into a single equation for $C=A B / \eta$, where $C \sim \eta^{2}$ for large $\eta$. For example, introducing the new variable $\rho=\log \eta$, and writing the derivative $\dot{C} / C$ of $\log C$ with respect to it as

$$
\frac{\dot{C}}{C}=\frac{\dot{A}}{A}+\frac{\dot{B}}{B}-1,
$$

we can eliminate $\dot{B} / B$ from both of (3.4), and then $\dot{A} / A$ can be eliminated from both the resulting equations to yield an equation for $\dot{C} / C$ in terms of $C$ of the form

$$
\left(\frac{\dot{C}}{C}+1\right)^{2}-\frac{3 C+1}{C}\left(\frac{\dot{C}}{C}+1\right)+\frac{3 C-4}{2 C^{2}}=0 .
$$

After some simplification this becomes

$$
\frac{\dot{C}}{C}=\frac{C+1}{2 C} \pm \frac{3}{2} \sqrt{1+\frac{1}{C^{2}}}
$$


where the positive root must be used so $\dot{C} \sim 2 C$ for large $C$, based on the properties of $C$ for large $\rho$ (or $\eta$ ). The resulting first-order nonlinear autonomous $\mathrm{ODE}$ for $C(\eta)$ can rearranged into

$$
\log \eta=\int \frac{2 d C}{C+1+3 \sqrt{C^{2}+1}}
$$

within an arbitrary constant. Potential branch-point singularities are apparent where $C= \pm i$.

\subsection{Change of independent variable}

Although an explicit anti-derivative of the integrand of (3.6) is not available, an implicit solution can be determined in terms of a new independent variable $w(\eta)$, in place of $C$, where

$$
C=w^{2}-\frac{1}{4 w^{2}} \text {. }
$$

This Joukowski-type transformation is chosen to match the far-field solution, so that $w \sim \eta$ for large $\eta$, and this ensures an appropriate periodicity. It also simplifies the evaluation of $A$ and $B$ across the entire $\eta$ plane, bearing in mind that $C$ is not an ideal choice of independent variable for any function (as for example it becomes large both as $\eta \rightarrow \infty$ and as $\eta \rightarrow 0$ ).

Replacing $C$ by $w$ allows (3.6) to be written as

$$
\log \eta=\int \frac{2\left(4 w^{4}+1\right) d w}{w\left(8 w^{4}+2 w^{2}+1\right)}
$$

which can be evaluated using partial fractions. The integrand of (3.8) signals the potential singular points in the solution, as singularities $w$ of $\eta(w)$ can occur wherever its derivative is zero or infinite. Among these are the four points $w_{1 \pm}^{2}=(-1 \pm i \sqrt{7}) / 8$ at which the denominator vanishes, given by

$$
w_{1 \pm}=\sqrt{\sqrt{2}-\frac{1}{2}} \pm i \sqrt{\sqrt{2}+\frac{1}{2}} \text { and }-w_{1 \pm} .
$$

It will be seen below that while $\pm w_{1 \pm}$ are not (finite) singular points of $A$ or $B$, they play a key role in their properties. Another potential singular point is at $w=0$, and there are four other candidate points where the numerator vanishes, at $w_{0 \pm}=(1 \pm i) / 2$ and $-w_{0 \pm}$. These latter four points correspond to $C= \pm i$, so that $\sqrt{C^{2}+1}=0$ as identified below (3.6).

Evaluating (3.8) gives that

$$
\eta=w^{2}\left(w^{2}-w_{1+}^{2}\right)^{\gamma_{+}}\left(w^{2}-w_{1-}^{2}\right)^{\gamma_{-}} \quad \text { where } \quad \gamma_{ \pm}=\frac{1}{4}(-1 \pm 3 i / \sqrt{7}),
$$

with the constant of integration chosen so that $\eta \sim w$ at large $w$. The factors in (3.9) must be evaluated so that no branch cuts extend to infinity in $w$ (since at $t=0$ there are no branch cuts in the complex plane for analytic initial data). To achieve this, the complex powers of the four factors $\left(w \pm w_{1 \pm}\right)$ are each evaluated using consistent argument ranges that preserve the symmetry of the far-field solution, for example using radial branch cuts through $w=0$. A well-defined and analytic function $\eta(w)$ can then be evaluated for any $w$ away from these branch cuts, along with a corresponding unique value of $C$ from (3.7) and $\eta(w)$ being one-to-one at larger values of $w$. The mapping is not bijective across that whole region, however, as there is the possibility of multiple values of $w$ (and hence $C$ ) at any given point $\eta$, located on different Riemann surfaces of $\eta(w)$. Prior to determining $A$ and $B$ in terms of $w$ it is therefore prudent to examine the transformation between $\eta$ (the original dependent variable) and $w$ in more detail, in particular to ensure that an appropriate $w$ domain is defined for a one-to-one relationship. 


\subsection{Properties of the transformation $\eta(w)$}

First, note that while $\eta \sim w$ is one-to-one for large $w$, near $w=0$ there are two possible values of $w$ for any given $\eta$, as $\eta \propto w^{2}$. As a result, only half of the points $w$ near the origin will be within the domain of the one-to-one mapping. Similar behaviour is anticipated near the four potential singular points at $w_{0 \pm}=(1 \pm i) / 2$ and $-w_{0 \pm}$ (where $d \eta / d w=0$ ), as locally $\eta$ will vary like $\left(w-w_{0 \pm}\right)^{2}$ for example. Within the disk of radius $|w|=1 / \sqrt{2}$, there are also the four branch points where $w_{1 \pm}^{2}=(-1 \pm i \sqrt{7}) / 8$ for which $\eta$ maps to infinity; for the given far-field conditions $\eta \sim w$ these points must be excluded from the desired domain. Combining this all suggests that one suitable $w$ domain for a one-to-one function $\eta(w)$ can have an almost 'clover-leaf pattern' within $|w| \leqslant 1 / \sqrt{2}$ as illustrated in Figure 1(a).

As a comparison with CBT, from (3.9) the point $\eta_{0}$ that corresponds with $w=w_{0+}=(1+i) / 2$ is at

$$
\eta_{0+}=\eta\left(w_{0+}\right)=i \sqrt{2}(1+i(4-\sqrt{7}))^{\gamma_{+}}(1+i(4+\sqrt{7}))^{\gamma_{-}} .
$$

Using Principal branches this value concurs with $\eta_{0}=0.7359+i 0.4920$, at (2.33) of CBT, to all 4 decimal places quoted. By conjugation $\eta_{0-}=\eta\left(w_{0-}\right)=\bar{\eta}_{0+}$ and the two other singularities corresponding to $-w_{0 \pm}$ are at $-\eta_{0+}$ and $-\eta_{0-}$. Note that changing the branches in any consistent manner that ensures the correct matching at large $w$ always yields one of these four $\eta$ values, for example adding integer multiples of $2 \pi i$ to the argument of each factor.

To proceed further it is convenient to fix the branch cuts in the $\eta$-plane. There are multiple choices, and some other possibilities are outlined in $\S 3.6$ below, but the simplest symmetry is to choose branch cuts that extend radially from $\eta=0$ to $\pm \eta_{0 \pm}$ in an 'X-shaped' formation, i.e. as given by $\eta= \pm \mu \eta_{0 \pm}$ for $0 \leqslant \mu \leqslant 1$. The boundary mapped out by these branch cuts in the $w$-plane could in principle be determined analytically, if implicitly, by seeking $w^{2}$ values in(3.9) for which $\eta=\mu \eta_{0+}$ for $0 \leqslant \mu \leqslant 1$; however, it was easier to calculate them numerically on either side of each cut and they map to a cloverleaf boundary shape in the $w$ domain, as illustrated in Figure 1(a).

The dots in Figure 1(a) are the mapped $\eta$-branch points $\eta= \pm \eta_{0 \pm}$, while the crosses are the four $w$-branch points at $w_{1 \pm}^{2}=(-1 \pm i \sqrt{7}) / 8$; at the latter points, $w$ is mapped to infinity in the $\eta$-plane. Since $\left|w_{1 \pm}\right|=2^{-3 / 4}$, these $w$-branch points are located slightly closer to the origin than the mapped $\eta$-branch points. Moreover, since $\pm w_{1 \pm}$ are all within the clover leaf, as expected they are outside of the domain required for a one-to-one mapping.

For illustration, surface plots of the real and imaginary parts of $\eta(w)$ in the $w$-plane, based on this $w$ domain, are shown in Figure 1(b)-(c) and note the distortion near the branch point at $w=0$.

Under some conditions (3.9) can also yield other possible $\eta$ singular points closer to the origin. For example, in $\S 3.6$ it will be seen that moving $w$ clockwise across the branch cut near $w_{1-}$ will effectively subtract $2 \pi$ from the argument of that factor (alone) and yield another potential singularity at

$$
\eta_{1+}=\eta\left(w_{0-}\right)=-i \sqrt{2}(1-i(4+\sqrt{7}))^{\gamma_{+}}(1-i(4-\sqrt{7}))^{\gamma_{-}} e^{-2 \pi i \gamma_{-}}=\eta_{0-} e^{-2 \pi i \gamma_{-}} .
$$

This point is rotated $\pi / 2$ from $\eta_{0_{-}}$, and its position at $\eta_{1+}=0.0829+i 0.1240$ concurs with that noted by CBT as being located on a different Riemann sheet. Correspondingly, there are three further potential singular points at $\bar{\eta}_{1},-\eta_{1}$ and $-\bar{\eta}_{1}$ based on similar effects near $w_{1+},-w_{1-}$ and $-w_{1+}$, respectively. It is significant that they only need to be considered if the $w$ domain contorts onto another Riemann sheet near either of the four $w$-branch points. Technically, it is also possible for (3.9) to yield other points that are further from the origin than $\eta_{0+}$ but they are not relevant under the far-field condition on $\eta(w)$. 
ON THE FORMATION OF CURVATURE SINGULARITIES IN VORTEX SHEETS
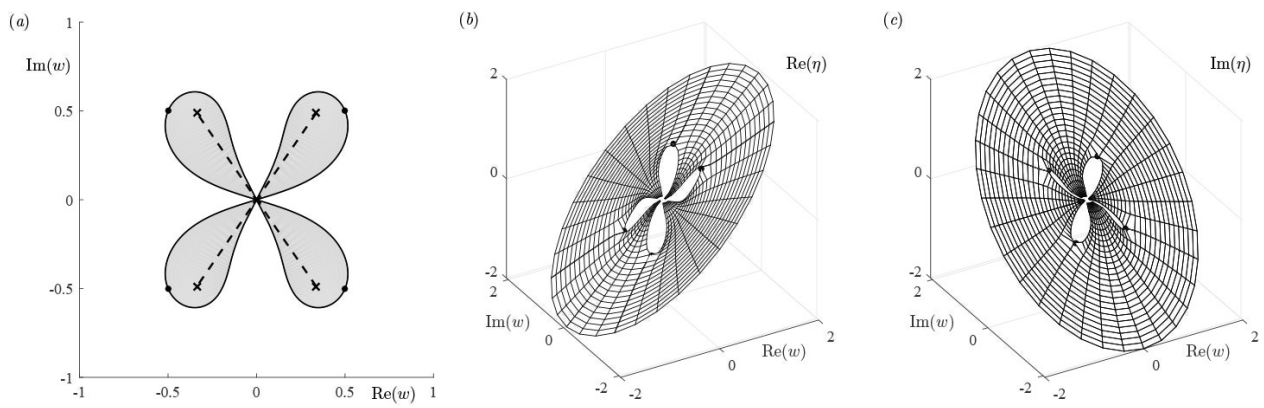

FIG. 1. (a) The domain of $\eta$ in terms of the transformed variable $w$, with the grey-shaded region excluded and showing the branch cuts from $\pm w_{1 \pm}$ that were used for most calculations in this paper. (b) Surface plot of the real part of $\eta(w)$ in the $w$-plane, matching with $\eta \sim w$ for large $w$ and showing the singular points at $\pm w_{0 \pm}$, and (c) the corresponding imaginary part of $\eta(w)$.

\subsection{Analytical expressions for $A$ and $B$}

Equations (3.4) can be written in terms of $\rho=\log \eta$ as

$$
\left(2-\frac{\dot{A}}{A}\right) \frac{\dot{B}}{B}=-\frac{1}{C} \quad \text { and } \quad \frac{\dot{A}}{A}\left(1-\frac{\dot{B}}{B}\right)=\frac{1}{C} \text {. }
$$

Taking the difference and using (3.5) gives that

$$
\frac{\dot{A}}{A}=\frac{2}{3}\left(\frac{\dot{C}}{C}+1\right)+\frac{2}{3 C}=\frac{C+1+\sqrt{C^{2}+1}}{C},
$$

and also that

$$
\frac{\dot{B}}{B}=\frac{1}{3}\left(\frac{\dot{C}}{C}+1\right)-\frac{2}{3 C}=\frac{C-1+\sqrt{C^{2}+1}}{2 C} .
$$

Using $C$ from (3.7), along with (3.8), to write these in terms of $w$ gives that

$$
\log A=\int \frac{8 w\left(4 w^{4}+1\right) d w}{\left(2 w^{2}-1\right)\left(8 w^{4}+2 w^{2}+1\right)} \quad \text { and } \quad \log B=\int \frac{4 w\left(4 w^{4}+1\right) d w}{\left(2 w^{2}+1\right)\left(8 w^{4}+2 w^{2}+1\right)},
$$

which again can be evaluated explicitly using partial fractions in terms of $w^{2}$.

Choosing the constant of integration to match with $A \sim w^{2}$ for $w \gg 1$ gives that

$$
A=\left(w^{2}-\frac{1}{2}\right)\left(w^{2}-w_{1+}^{2}\right)^{\alpha_{+}}\left(w^{2}-w_{1-}^{2}\right)^{\alpha_{-}} \quad \text { and } \quad \alpha_{ \pm}= \pm i / \sqrt{7},
$$

and ensuring $B \sim w$ for $w \gg 1$ gives

$$
B=\left(w^{2}+\frac{1}{2}\right)\left(w^{2}-w_{1+}^{2}\right)^{\beta_{+}}\left(w^{2}-w_{1-}^{2}\right)^{\beta_{-}} \quad \text { and } \quad \beta_{ \pm}=-\frac{1}{4}(1 \pm i / \sqrt{7}) .
$$

As for (3.9), these expressions are evaluated within the $w$ domain by using individual linear factors within each complex power, and so that branch cuts do not extend to infinity in the $w$-plane. Here $A$ at large $w$ repeats when $\arg (w)$ is increased by $\pi$, whereas (like $\eta$ ) the function $B$ has period $2 \pi$. How $A$ and $B$ behave for smaller $w$ depends on the domain used, and hence the choice of branch cuts in $\eta$. 
Given that $w$ is discontinuous across the excluded regions of the $w$-plane, for example across the clover-leaf boundary in Figure 1 in the case of radial branch cuts in the $\eta$-plane, both $A$ and $B$ are expected to have discontinuities across these four branch cuts as well. This is illustrated in Figures 2 and 3, which show plots of the real and imaginary parts of $\left(A-\eta^{2}+\frac{1}{2}\right)$ and $(B-\eta)$, respectively. The far-field behaviour is removed, to make their features more evident, and as expected from (2.29) in CBT both functions approach constants at infinity. In all plots the solution is finite everywhere and typically discontinuous across branch cuts, although $\operatorname{Im}\left(A-\eta^{2}\right)$ is continuous with discontinuous derivative.
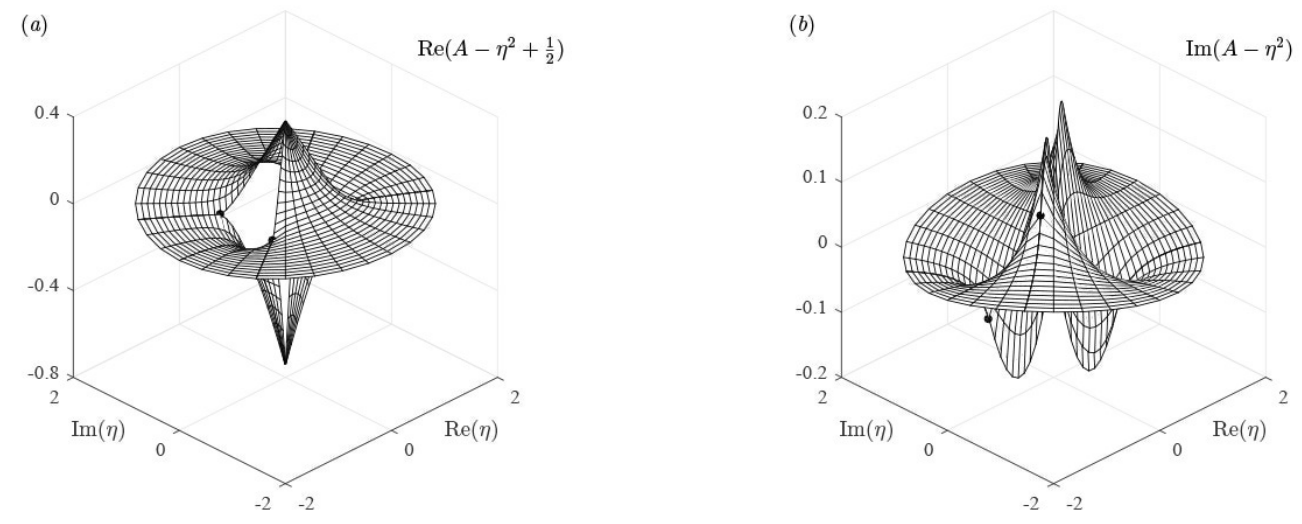

FIG. 2. Surface plots of the real and imaginary parts of $\left(A-\eta^{2}+\frac{1}{2}\right)$ in the $\eta$-plane, showing the four singular points at $\eta_{0}, \bar{\eta}_{0}$, $-\eta_{0}$ and $-\bar{\eta}_{0}$ based on the radial branch cuts used here.
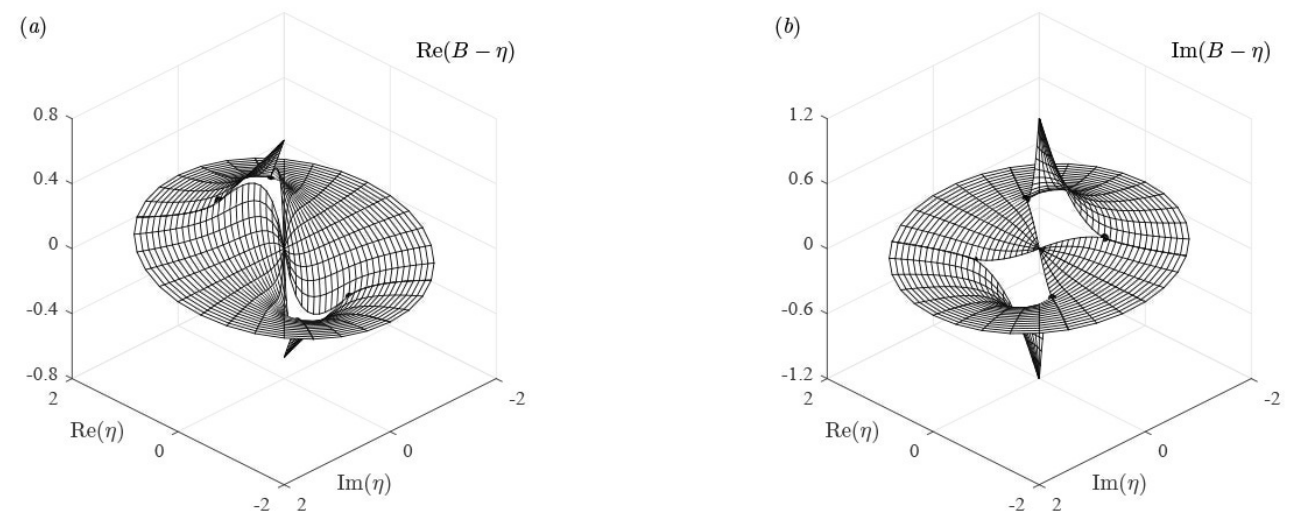

FIG. 3. Corresponding surface plots to Figure 2 for the real and imaginary part of $(B-\eta)$ in the $\eta$-plane.

Other configurations of branch cuts for $A$ and $B$ can be used in the $\eta$-plane, for example extending from $\eta_{0-}$ to $\eta_{0+}$ and from $-\eta_{0-}$ to $-\eta_{0+}$, and some options for this are outlined in $\S 3.6$. 


\subsection{Properties of $A$ and $B$}

Although $C$ is an analytic function of $w$ near $\pm w_{0 \pm}$, and $A$ and $B$ are also analytic functions of $w$ away from the branch cuts that extend from $\pm w_{0 \pm}$, it is apparent above that each of the points $\pm w_{0 \pm}$ are singularities of $w(\eta)$. Expanding (3.8) about any such chosen point $w_{0}= \pm w_{0 \pm}$ then (3.8) implies that

$$
\frac{\eta-\eta_{0}}{\eta_{0}} \sim \frac{16 w_{0}^{2}\left(w-w_{0}\right)^{2}}{8 w_{0}^{4}+2 w_{0}^{2}+1}+O\left(w-w_{0}\right)^{3} .
$$

where $\eta_{0}= \pm \eta_{0 \pm}$ is the corresponding $\eta$ value. As a result, each of the four branch points $\eta_{0}= \pm \eta_{0 \pm}$ of $w(\eta)$ corresponds to a $\frac{1}{2}$-power singularity in $\left(\eta-\eta_{0}\right)$. It follows that $C, A$ and $B$ are all potentially singular functions of $\eta$ at those branch points as well.

A simple way to clarify the form of these singularities in $A$ and $B$ is to write (3.6) for $C(\eta)$ as

$$
\eta C_{\eta} \sim \frac{1}{2}\left(C_{0}+1\right)+\frac{3}{2} \sqrt{2 C_{0}\left(C-C_{0}\right)}+O\left(C-C_{0}\right)
$$

near each branch point $\eta=\eta_{0}$, where $C_{0}= \pm i$ is the corresponding $C$ value. Upon integration locally this gives that

$$
\left(C-C_{0}\right) \sim \frac{1}{2}\left(C_{0}+1\right)\left(\eta-\eta_{0}\right) / \eta_{0}+\sqrt{C_{0}\left(C_{0}+1\right)\left(\eta-\eta_{0}\right)^{3} / \eta_{0}^{3}}+O\left(\eta-\eta_{0}\right)^{2}
$$

near $\eta_{0}$ and hence that $C(\eta)$ has a $\frac{3}{2}$-power singularity in $\left(\eta-\eta_{0}\right)$.

The local forms of $A$ and $B$ can be deduced in a similar manner, using their expansions near the same points $\eta_{0}$. For example, expanding $A$ in (3.11) first near $C_{0}$ and then in terms $\eta$ using (3.15) gives that

$$
\frac{\eta A_{\eta}}{A}=\frac{C+1+\sqrt{C^{2}+1}}{C} \sim \frac{C_{0}+1+\sqrt{C_{0}\left(C_{0}+1\right)\left(\eta-\eta_{0}\right) / \eta_{0}}}{C_{0}}+O\left(\eta-\eta_{0}\right) .
$$

By evaluating (3.13) at the four singular points $w_{0}= \pm w_{0 \pm}$ it is clear that $A$ exists and is non-zero at each point $\eta_{0}$, and further that from (3.16) that its first derivative $A_{\eta}$ exists and is finite there also. Integrating (3.16) locally, as for (3.15), yields that $A$ has a non-zero $\frac{3}{2}$-power singularity in $\left(\eta-\eta_{0}\right)$. The argument for $B$ is similar.

More broadly, $A_{\eta}$ can be expressed in terms of $w$ using (3.16) with (3.7), (3.9) and (3.13) and from that it is apparent that $\left|A_{\eta}\right|$ is both non-zero and finite everywhere in any relevant $w$ domain, away from $\pm w_{1 \pm}$. The value of $\left(1+s_{\xi}\right)$ based on (3.2a) therefore becomes non-zero for all $\eta$ once $t>0$, and the initial singularity in the first equation in (2.1) immediately vanishes. A similar argument applies to $\left|B_{\eta}\right|$ and the second equation in (2.1).

\subsection{Other options for branch cuts}

Radially-aligned branch cuts between $w=0$ and each of $\pm w_{1 \pm}$, as used to represent $\eta, A$ and $B$ in terms of $w$ above, are arguably the simplest symmetric representation in terms of $\zeta=\eta \omega t^{1 / 2}$ as the radius increases with time $t$. However, they are not the only option for single-valued solutions, and other symmetric branch-cut locations for $A$ and $B$ in terms of $\eta$ include the 'H-shaped' (dotted) configuration in Figure 4(a). Here the cuts extend between adjacent pairs of the branch points $\pm \eta_{0 \pm}$, with a third cut between those lines (ensuring that there are no $w$ branch cuts extending to infinity). Alternatively, 'N-shaped' cuts can be used, illustrated in Figure 4(a) (dash-dotted) using branch cuts along circular arcs. Either of these options align with the periodicity noted in CBT, and the corresponding $w$ domains 

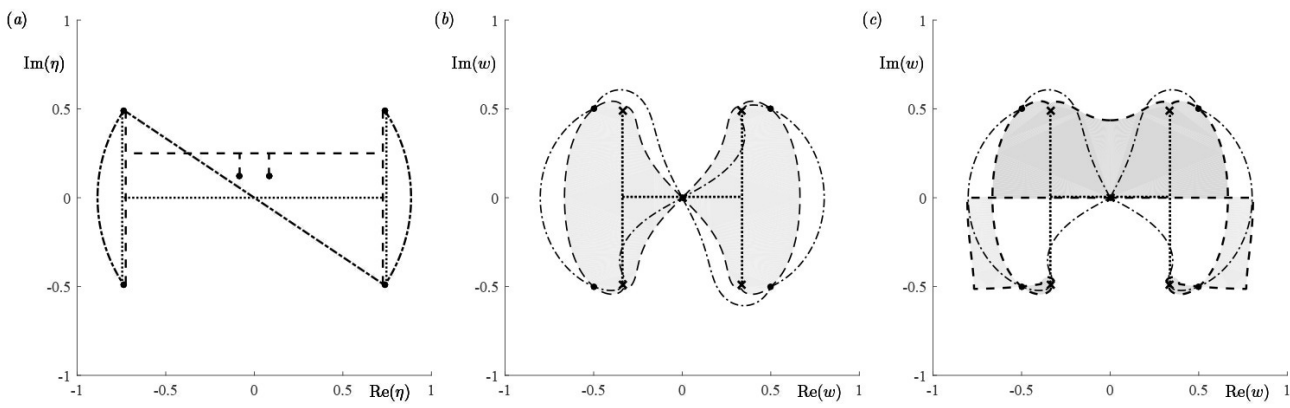

FIG. 4. (a) Alternative branch-cut locations in the $\eta$ plane for $A$ and $B$, as discussed in $\S 3.6$. (b) The 'H-shaped' $\eta(w)$ domain (dashed, with grey-shaded region excluded) as well as the domain boundary for ' $\mathrm{N}$-shaped' cuts (dash-dotted). Typical $w$-branch cuts used with (3.8) between $w=0$ and $\pm w_{1 \pm}$ are also shown (dotted). (c) Similar but for rotated 'E-shaped' cuts in $\eta$ (dashed, with dark grey-shaded regions excluded), and also the $w$ boundary for curved 'M-shaped' cuts that join at $\eta=0$ (dash-dotted).

are illustrated in Figure 4(b). Although these domain boundaries appear quite close to some of $\pm w_{1 \pm}$, those points all lie outside the $w$ domain. For the ' $\mathrm{N}$-shaped' case the domain boundary extends across the illustrated $w$ branch cuts (shown dotted), but this can be avoided using curved cuts in $w$.

Other options with the same symmetry include 'I-shaped' and 'Z-shaped' configurations based on pairs of horizontal cuts (with a third cut between them) and the $w$ domains for these are broadly similar to those in Figure 4(b) but rotated by $90^{\circ}$.

Breaking from the symmetry implied in CBT, for example by raising the 'cross-bar' of the ' $\mathrm{H}$ shaped' cut (shown dashed) in Figure 4(a) so it lies above $\operatorname{Im}\left(\eta_{1+}\right)=0.12$, reveals additional singular points at $\eta_{1+}$ and $-\eta_{1-}$. The $w$ domain corresponding to the resulting rotated 'E-shaped' branch cuts is shown in Figure 4(c) when $\operatorname{Im}(\eta)=0.15$ along the 'cross-bar'. These $w$ values are not on a single Riemann surface, as the domain extends under the branch cuts and into the lightly-shaded region (the width of which increases as the 'cross-bar' is raised further). In the shaded region near $w_{1-}$ the argument of $\left(w-w_{1-}\right)$ is $2 \pi i$ less than in the central part of the domain, and hence $w_{0-}$ maps to $\eta_{1+}$ rather than $\eta_{0-}$. Similarly, near $-w_{1+}$ an increase by $2 \pi i$ in the argument of $\left(w+w_{1+}\right)$ maps $-w_{0+}$ on that sheet to $-\eta_{1-}$. These are precisely the two points observed by CBT when integrating numerically from $\eta=0-i L$. Similar effects occur if the cross-bar of an 'H-shaped' cut is lowered, but with singular points at $\eta_{1-}$ and $-\eta_{1+}$ sintead. Note, however, that such additional branch points do not arise for the 'I-shaped' 'I-shaped' or 'Z-shaped' configurations, as for example the change in argument across the cut near $w_{1-}$ has the opposite sign and so $w_{0-}$ is mapped outwards.

The domain for curved 'M-shaped' cuts in Figure 4(c) likewise breaks the quadrant-based periodicity noted by CBT, but avoids $\eta_{1+}$ and $-\eta_{1-}$, as would a hybrid of ' $\mathrm{H}$ ' and 'M-shaped' cuts that has no symmetries at all. That said, a symmetrical orientation of the cuts is arguably the most obvious choice in the absence of external factors.

In Figure 1(a) the excluded region of the $w$ domain near the origin represents half of the local area, with the four 'clover leaves' subtending an angle of $45^{\circ}$ at $w=0$. In contrast, both cases in Figure 4(b) have two excluded regions, each subtending $90^{\circ}$ at $w=0$. These all ensure $\eta(w)$ is one-to-one locally, bearing in mind that $\eta \propto w^{2}$ near $w=0$. A third possibility is illustrated by the rotated 'E-shaped' branch cuts in Figure 4(c), where a single region with $\operatorname{Im}(w)<0$ subtends $180^{\circ}$ at $w=0$. It is relevant to observe here that, while $w=0$ is a singularity for $\eta(w)$, both $A(\eta)$ or $B(\eta)$ are regular at its image point $\eta=0$ when branch cuts are arranged to avoid that. 
Despite these various changes to the $w$ domains of $A$ and $B$ within the rectangular region bounded by $\pm \eta_{0 \pm}$, their main features are as can be expected from their plots in Figures 2 and 3. In particular, $A$ and $B$ each have discontinuities in values or gradients along the chosen $\eta$ branch cuts but otherwise their values are bounded and the altered features are unremarkable.

\section{Exact solution for singularity formation: other analytic cases}

CBT also considered functions $s_{0}$ and $s_{0}^{*}$ with both $s_{0 \xi}\left(\xi_{0}\right)=-1$ and $s_{0 \xi}^{*}\left(\xi_{0}\right)=-1$ and performed a similar type of small-time expansion about $\xi_{0}$. They assumed that the $O\left(\zeta^{2}\right)$ term in both $s_{0}$ and $s_{0}^{*}$ were non-zero, arguably the most common case, but with little effort a more general exact analysis can be formulated. As a result, here it is assumed that the functions $s_{0}$ and $s_{0}^{*}$ are both analytic at $\xi_{0}$ but with first non-linear terms that are $O\left(\zeta^{m}\right)$ and $O\left(\zeta^{n}\right)$, respectively, where $m \geqslant 2$ and $n \geqslant 2$ are integers.

Again following the approach of CBT, series solutions for $s(\xi, t)$ and $s^{*}(\xi, t)$ at small values of $\zeta=\xi-\xi_{0}$ and $t$ are sought of the form

$$
\begin{aligned}
& s=s_{00}-\zeta+\frac{1}{m !} s_{0 m} \zeta^{m}+\cdots-\left[\frac{(n-1) !\left(1+\sigma_{01}\right)}{2 s_{0 n}^{*} \zeta^{n-1}}+\ldots\right] t+\ldots, \\
& s^{*}=s_{00}^{*}-\zeta+\frac{1}{n !} s_{0 n}^{*} \zeta^{n}+\cdots+\left[\frac{(m-1) !\left(1+\sigma_{01}\right)}{2 s_{0 m} \zeta^{m-1}}+\ldots\right] t+\ldots,
\end{aligned}
$$

As for (3.1a) and (3.1b), this expansion becomes 'disordered' once $\zeta^{k} \sim t$, where $k=m+n-1$, or when $\zeta=O\left(t^{1 / k}\right)$. A 'similarity' variable $\eta=\zeta / \Omega t^{1 / k}$ is then suggested in terms of

$$
\Omega=\left(\frac{k m ! n !\left(1+\sigma_{01}\right)}{2 s_{0 m} s_{0 n}^{*}}\right)^{1 / k},
$$

noting that $\Omega$ here differs slightly from that used by CBT when $m=n=2$, by a factor of $(3 / 2)^{1 / 3}$. A small-time expansion of $s$ and $s^{*}$ in terms of $\eta$ and $t$ can proceed in a similar form to (3.2a), with

$$
\begin{aligned}
s & =s_{00}-\eta \Omega t^{1 / k}+\frac{1}{m !} s_{0 m} \Omega^{m} A(\eta) t^{m / k}+\ldots, \\
\text { and } \quad s^{*} & =s_{00}^{*}-\eta \Omega t^{1 / k}+\frac{1}{n !} s_{0 n}^{*} \Omega^{n} B(\eta) t^{n / k}+\ldots
\end{aligned}
$$

Both $m / k$ and $n / k$ are strictly less than one so $O(t)$ terms arising from (2.1) do not affect this leadingorder representation. Substituting (4.2a) and (4.2b) into (2.1) for small $t$ yields the first-order ODEs

$$
m A-\eta A_{\eta}=-1 / B_{\eta} \quad \text { and } \quad n B-\eta B_{\eta}=1 / A_{\eta},
$$

and matching for large $\eta$ requires that $A \sim \eta^{m}$ and $B \sim \eta^{n}$. As for $\S 3.1$, these can be combined into a single equation for $C=A B / \eta$, and here the derivative $\dot{C}$ of $C$ with respect to $\rho=\log \eta$ satisfies

$$
\dot{C}=\frac{m+n-2}{2} C+\frac{m-n}{m n}+\frac{m+n}{2} \sqrt{C^{2}+4 a^{2}}, \text { where } a=1 / m n .
$$

The positive root is used so $\dot{C} \sim 2 C$ for large $C$, since $C \sim \eta^{k}$ for large $\eta$.

Potential branch-point singularities are apparent here when $C= \pm 2 a i$. As in $\S 3.2$, an implicit solution can be determined in terms of a new independent variable $w$, in place of $C$, where in this case

$$
C=w^{k}-a^{2} / w^{k} .
$$


Replacing $C$ by $w$ allows (4.4) to be written as

$$
\log \eta=\int \frac{k\left(w^{2 k}+a^{2}\right) d w}{w\left(k w^{2 k}+(m-n) a w^{k}+a^{2}\right)},
$$

so that it can be evaluated explicitly using partial fractions. It follows that $2 k$ of the potential singularities of $\eta(w)$ in the $w$-plane are at the $k$ complex roots of each of $w_{0 \pm}^{k}= \pm a i$ where the numerator vanishes; as in $\S 3$, these lie on the boundary of the required domain. In addition, the denominator of (4.6) vanishes at both $w=0$ and the $2 k$ branch points $w$ with

$$
w^{k}=w_{1 \pm}^{k}=\frac{a}{2 k}(-(m-n) \pm \Delta) \quad \text { where } \quad \Delta=\sqrt{(m-n)^{2}-4 k}
$$

Again, these latter points lie outside the domain of $\eta(w)$ necessary for a one-to-one relationship but, unlike in the $k=2$ case here, $\Delta$ may be real-valued if $|m-n|$ is sufficiently large.

A solution of (4.6) with $\eta \sim w$ when $w \gg 1$ can be found by integration of (4.6), leading to

$$
\eta=w^{k}\left(w^{k}-w_{1+}^{k}\right)^{\gamma_{+}}\left(w^{k}-w_{1-}^{k}\right)^{\gamma_{-}} \quad \text { where } \quad \gamma_{ \pm}=\frac{1-k}{2 k} \mp \frac{m^{2}-n^{2}}{2 k \Delta}
$$

where powers are evaluated so that no branch cuts extend to infinity. Cuts may be directed radially inwards towards $w=0$, or otherwise some of the cases in $\S 3.6$ can be generalised. Each term with a complex power can be written as a product of $k$ linear factors prior to evaluating (4.7), to ensure appropriate argument choices, and that $\eta$ matches the large $w$ condition.

The corresponding points to $w^{k}=w_{0+}^{k}=i a$ in the $\eta$-plane are at

$$
\eta_{0}=2 i k(a / 2 k)^{1 / k}((m-n)-\Delta+2 i k)^{\gamma_{+}}((m-n)+\Delta+2 i k)^{\gamma_{-}}
$$

and at multiples of $\exp (2 \pi i / k)$ times that, with the $k$ points corresponding to $w_{0-}^{k}$ at their conjugates. Analogues of the $\eta_{1}$ values are also conceivable for non-symmetric branch cuts.

Following a similar approach to $\S 3.3$, expressions for $A$ and $B$ in this case can be obtained as

$$
\log A=\int \frac{m k w^{k-1}\left(w^{2 k}+a^{2}\right) d w}{\left(w^{k}-a\right)\left(k w^{2 k}+(m-n) a w^{k}+a^{2}\right)}
$$

and

$$
\log B=\int \frac{n k w^{k-1}\left(w^{2 k}+a^{2}\right) d w}{\left(w^{k}+a\right)\left(k w^{2 k}+(m-n) a w^{k}+a^{2}\right)} .
$$

These may be evaluated using partial fractions in terms of $w^{k}$ and, selecting the constants of integration so that $A \sim w^{m}$ and $B \sim w^{n}$ for $w \gg 1$, this gives

$$
A=\left(w^{k}-a\right)\left(w^{k}-w_{1+}^{k}\right)^{\alpha_{+}}\left(w^{k}-w_{1-}^{k}\right)^{\alpha_{-}} \quad \text { for } \quad \alpha_{ \pm}=\frac{1-n}{2 k} \mp \frac{2 m^{2}+m n+n^{2}-3 k-1}{2 k \Delta},
$$

along with

$$
B=\left(w^{k}+a\right)\left(w^{k}-w_{1+}^{k}\right)^{\beta_{+}}\left(w^{k}-w_{1-}^{k}\right)^{\beta_{-}} \quad \text { for } \quad \beta_{ \pm}=\frac{1-m}{2 k} \pm \frac{m^{2}+m n+2 n^{2}-3 k-1}{2 k \Delta} .
$$


The same $w$ domain as described above for (4.7) is used with (4.11), with each of the complex powers evaluated using a product of individual linear factors. As noted in $\S 3.6$, in principle there is no reason why the $\eta$ branch cuts need extend radially from $\eta=0$, nor that the branch cuts in $w$ need be straight lines. A plot of the $w$ domain is not given here, but for $m=n=2$ it broadly looks like Figure 1(a) but with $2 k=6$ excluded 'clover leaves' rather than four.

Properties of $A$ and $B$ for integers $m \geqslant 2$ and $n \geqslant 2$ follow in a similar way to $\S 3$, including that each have $2 k$ singular points in the $\eta$-plane, with associated branch cuts. The branch points are given by $\eta_{0}$ in (4.8) and its conjugate, with a periodicity of $k$ from both. For larger values of $\eta$ the features of $A$ and $B$ are dominated by their boundary condition at large $\eta$, with $A \sim \eta^{m}$ and $B \sim \eta^{n}$ and hence an $m$ and $n$-fold periodicity in the far field respectively. Higher-order terms in the expansions of the solutions for large $\eta$ will proceed in relative powers of $\eta^{k}$, in accordance with the dependencies on $w^{k}$ in (4.11) and (4.12) and that $\eta \sim w$ for large $w$.

As for the $k=2$ case in $\S 3$, the solutions near each of the branch points have $\frac{3}{2}$-power singularities, since the square root term in (4.4) is always present and the transformation $\eta(w)$ is always locally quadratic in $\left(w-w_{0}\right)$ near each singular point $w_{0}$. In fact, when evaluated at $m=2$ and $n=1$ the solutions here are identical to those in $\S 3$, so it is only the differing details of the expansions (3.2a) and (3.2b) compared with (4.2a) and (4.2b), that requires $m \geqslant 2$ and $n \geqslant 2$ here.

As a further point of comparison, the $m=n=2$ case was considered in $\S 2.4 .2$ of CBT, and the properties of the solutions above for $k=3$ match with the symmetry conditions that they list below their (2.39). That said, the singular points from (4.8) here give that $\eta_{0}=3^{1 / 3} \exp (\pi i / 6) / 2$, with the other five singular points spaced by multiples of $\exp (\pi i / 3)$. As a slightly different scaling has been used here from CBT, the equivalent location of $\eta_{0}$ in their scaling is $(3 / 4)^{2 / 3} \exp (\pi i / 6)$ and so the right-hand side of their (2.40) should be $-81 / 256 \approx-0.3164$. The value given in CBT can be verified to be a typographical error by using their numerical approach (and indeed the number in (2.40) happens to be a sixth root of their $\left.\left|\eta_{0}\right|\right)$.

\section{Remarks}

We have considered solutions to the nonlinear Kelvin-Helmholtz model for initial data such that the problem is well-posed for a finite time. For such initial data, solutions can develop a physical singularity after a finite time. Depending on the initial condition, singularities of varying forms can develop on the interface, e.g. Duchon \& Robert (1988); Caflisch \& Orellana (1986); Caflisch \& Semmes (1990). However, for a broad class of initial data it is a $\frac{3}{2}$-power curvature singularity that is observed numerically. Since a local analysis near the singularity allows $q$-power singularities for $1<q<2$, the question arises why it is $q=\frac{3}{2}$ that is so often observed; the local linear analysis near the singularity yields no hint. CBT argued that the selection is determined at $t=0+$, when $\frac{3}{2}$-power singularities can spontaneously form in the complex plane away from the real physical axis (by a nonlinear process). However, CBT's analysis depended on the numerical solution of certain ODEs.

We have revisited CBT's analysis, and solved the relevant ODEs exactly. Our analysis confirms the character of the $\frac{3}{2}$-power singularity in $s$ and $s^{*}$, together with the number and precise locations of the branch points. Further, the exact solutions demonstrate that there are no other singular points in $\eta$ for any symmetric choice of branch cuts, although a small number of additional singular points $\eta_{1}$ can be present for more convoluted branch cuts. The same analytical approach enables these results to be generalised to a wider class of analytic initial conditions than considered by CBT; yet again $\frac{3}{2}$ power singularities spontaneously form at $t=0+$. In addition, the analytical solution also demonstrates that, having initially formed $\frac{3}{2}$-power singularities at points where $\left(1+s_{\xi}\right)$ and/or $\left(1+s_{\xi}^{*}\right)$ are zero, 
the denominators of (2.1) immediately become non-zero locally once $t>0$; thereafter those singular solutions are expected to move around in the complex plane, as also apparently happens with other $q$-power singularities with $1<q<2$ where those are present in the initial conditions.

An aspect of the solution that we have not investigated in detail is the time evolution of the branch cuts. In order to match as $\eta \rightarrow \infty$, the solution is required to be single valued, and so the cuts do not extend to infinity (or at least not across the far-distant real $\xi$ axis). The main results shown in this paper are illustrated using radial branch cuts, but using cuts parallel with the real or imaginary $\eta$-axes is also simple to implement (although less arguable, as the orientation of the $\eta$-axes relative to the $\xi$-axes depends on an unknown constant). More generally, however, the cuts can be curved, and for $t=O(1)$ might be expected to evolve in time; although a constraint is that they should not intersect with the real axis in advance of the singularities.

Similar assumptions to CBT have been used here, but more generally it is conceivable that the solutions in $\S 4$ could be applied to non-integer values of $m$ and/or $n$ in an initial condition for which $\left(1+s_{\xi}\right)$ and/or $\left(1+s_{\xi}^{*}\right)$ are zero, including fractional and perhaps irrational values. Higher-order terms in the small-time expansions might also be considered, although it is probably unlikely that these will shed significant further light on the problem (or yield exact analytical solutions).

The key implication of our exact solutions is that for a wide range of initial conditions $\frac{3}{2}$-power singularities, and only $\frac{3}{2}$-power singularities, spontaneously form at $t=0+$ for analytic initial data.

An important question remaining is how the vortex sheet evolves for times after the singularity has formed on the real axis, i.e. for $t>t_{s}$. In order to consider this issue, the Birkhoff-Rott model needs to be 'regularized', e.g. by introducing viscosity, surface tension, so-called 'blobs' or a finite-width to the vortex sheet. How these regularization change the formation of the singularities at $t=0+$ is the subject of current research.

\section{REFERENCES}

Birkhoff, G. (1962) Helmholtz and Taylor instability. Proc. Symp. Appl. Math. Am. Math. Soc., 13, 55-76.

CAflisch, R. E. \& ORellana, O. F. (1986) Long term existence for slightly perturbed vortex sheet. Commun. Pure Appl. Maths, 39, 1-18.

Caflisch, R. E. \& Orellana, O. F. (1989) Singular solutions and ill-posedness for the evolution of vortex sheets. SIAM J. Math. Anal., 20, 293-307.

CAflisch, R. E. \& SEmmes, S. (1990) A nonlinear approximation for vortex sheet evolution and singularity formation. Physica D, 41, 197-207.

Cowley, S. J., BAKer, G. R., \& TAnVeer, S. (1999) On the formation of Moore curvature singularities in vortex sheets. J. Fluid Mech., 378, 233-267, referred to as CBT herein.

Duchon, J. \& Robert, O. (1988) Global vortex-sheet solutions of Euler equations in the plane. J. Diff. Equat., 73, 215-224.

KrASNY, R. (1986) A study of singularity formation in a vortex sheet by the point-vortex method. J Fluid Mech. 167, 65-93.

Meiron, D. L., Baker, G. R., \& Orszag, S.A. (1982) Analytic structure of vortex sheet dynamics. Part 1. Kelvin-Helmholtz instability J. Fluid Mech., 114, 283-298.

Moore, D. W. (1979) The spontaneous appearance of a singularity in the shape of an evolving vortex sheet. Proc. Roy. Soc. Lond. A 365, 105-119.

Moore, D. W. (1985) Numerical and analytical aspects of Helmholtz instability. In Theoretical and Applied Mechanics, Proc. XVIth ICTAM (ed. F. I. Niordson \& N. Olhoff), 263-274. North-Holland.

Pugh, D. A. (1989) Development of vortex sheets in Boussinesq flows - formation of singularities. PhD Thesis, University of London. 
Saffman, P. G. \& BAKer, G. R. (1979) Vortex interactions. Ann. Rev. Fluid Mech. 11, 95-122.

Sulem, P., Sulum, C., Bardos, C. \& Frisch, U. (1981) Finite time analyticity for the two and three dimensional Kelvin-Helmholz instability. Commun. Math. Phys. 80, 485-516. 